\title{
La acción térmica del mate como factor de riesgo en el cáncer bucal
}

María Mercedes González Prof. Titular Cátedra Clínica Estomatológica. Facultad de Odontología. Universidad Nacional del Nordeste. E-mail:mmgonzalez53@gmail.com.

Estefanía Raquel Fernández Auxiliar Docente de Primera Categoría Cátedra Clínica Estomatológica. Facultad de Odontología. Universidad Nacional del Nordeste

Gabriela G. Bessone Prof. Adjunta Cátedra Introducción a la Odontología. Facultad de Odontología. Universidad Nacional del Nordeste

Carlos Alejandro Rosales Becario de Iniciación en Investigación. Facultad de Odontologia. Universidad Nacional del Nordeste

Lugar de Trabajo: Facultad de Odontología. Avenida Libertad 5450.

3400 Corrientes.

Tel.: 03794457992

\section{Resumen}

El presente trabajo de investigación fue realizado en la Facultad de Odontología de la Universidad Nacional del Nordeste. Se propuso determinar la acción térmica del mate como factor de riesgo en el cáncer bucal. Se trabajó con 100 individuos que concurrieron voluntariamente a la Cátedra Clínica Estomatológica, al Servicio de Estomatología y Hospital Odontológico Universitario, cuyo rango de edades oscilaron entre 20 y 60 años, de ambos sexos, con antecedentes de consumo de mate y mate y tabaco, conformando dos grupos de trabajo. El trabajo consistió en el registro de temperatura y toma de muestras a través de citología exfoliativa de las mucosas bucales antes y después del consumo de mate. Se enviaron al Laboratorio de Anatomía Patológica para su posterior estudio histopatológico. Los resultados demostraron que no existen diferencias significativas en cuanto al sexo. El rango de edades más afectado fue de 20 a 31 años. Se demostró que a mayor temperatura del agua ingerida durante el consumo de mate, mayores son las temperaturas registradas en las mucosas bucales estudiadas, provocando alteraciones epiteliales en las mismas. Se observó que la zona topográfica afectada en mayor grado es el paladar duro, principalmente en pacientes fumadores, en los cuales se evidenciaron alteraciones celulares inflamatorias severas y moderadas. 
REVISTA FACULTAD DE ODONTOLOGÍA

ISSN No 1668-7280 - Vol. IX No 1 - 2016

14
INVESTIGACIÓN

González - Fernández - Bessone - Rosales
Palabras Clave

Infusión, temperatura, metaplasia epitelial, neoplasias.

\section{Summary}

This research was conducted at the Faculty of Dentistry at the National University of the Northeast. It was proposed to determine the mate as a risk factor predisposing oral cancer. We worked with 100 individuals who attended voluntarily and Dental University Hospital, whose range of ages ranged between 20 and 60 years, of both sexes, with a history of consumption of mate and mate and snuff, forming two working groups Stomatology Service. The work consisted of recording temperature and sampling through exfoliative cytology of oral mucosa before and after consumption of matte. They were sent to the Laboratory of Pathology for histopathological study. The results showed no significant differences in gender. The most affected age range was 20 to 31 years. It was shown that the higher the temperature of water ingested during mate consumption, the higher the temperatures in the studied oral mucosa, causing epithelial alterations in them. It was noted that the topographic greater extent affected area is the hard palate, especially in smokers, in whom severe and moderate inflammatory cellular alterations were evident.

\section{Key Words}

Infusion, temperature, epithelial metaplasia, neoplasias.

\section{Sumário}

Esta pesquisa foi realizada na Faculdade de Odontologia da Universidade Nacional do Nordeste. Foi proposto para determinar a ação térmica do mate como um fator de risco para o cancro oral. Nós trabalhamos com 100 indivíduos que participaram voluntariamente com a Clínica Dental Chair no Hospital e University Dental, cuja gama de idades variaram entre 20 e 60 anos, de ambos os sexos, com história de consumo de mate e erva-mate e rapé Estomatologia Serviço, formando dois grupos de trabalho. $O$ trabalho consistiu de temperatura registo e de amostragem através de citologia esfoliativa da mucosa oral antes e após o consumo de mate. Eles foram enviados para o Laboratório de $\mathrm{Pa}$ tologia para estudo histopatológico. Os resultados não mostraram diferenças significativas em gênero. A faixa etária mais acometida foi de $20 \mathrm{a}$ $3 \mathrm{I}$ anos. Foi mostrado que quanto maior a temperatura da água ingerida durante o consumo imediato, maior a temperaturas na mucosa oral estudados, causando alterações epiteliais neles. Notou-se que o topográfico maior medida área afetada é o palato duro, especialmente em fumantes, nos quais alterações celulares inflamatórias graves e moderados foram evidentes.

\section{Palavras Chave}

Infusão, temperatura, metaplasia epitelial, neoplasias.

\section{Introducción}

El cáncer bucal es un problema de salud que afecta un grupo significativo de personas en todo el mundo, capaz de producir notables secuelas anatómicas y fisiológicas en quienes lo padecen. La incidencia, la prevalencia y la severidad de esta enfermedad varían de un país a otro.

Numerosos estudios han demostrado que la cancerización es un fenómeno relativamente complejo en el que actúan o participan varios factores y donde probablemente ninguno de ellos por sí solo, sea capaz de originar una neoplasia. Esto justifica el criterio de que el cáncer es una enfermedad de causa multifactorial, que depende de la acción de factores sociales o conductuales, hereditarios y ambientales.

Un grupo de investigadores han desarrollado un estudio sobre el consumo de la yerba mate, el efecto carcinogénico de esta bebida y los impactos que causan sus riesgos.

La yerba mate es una bebida consumida habitualmente en América del Sur y entre los sudamericanos en todo el mundo. Es elaborado a partir de hojas secas y stemlets de un árbol perenne el llex paraguayensis (yerba mate), una especie que pertenece a la familia Auilofoliaceae'.

Alrededor de 300.000 toneladas de yerba mate son cosechadas anualmente en Sudaméri$\mathrm{ca}$, donde es consumida fundamentalmente en Argentina, Uruguay, Paraguay y el sur de Brasil. 
Pero su consumo se ha ido extendiendo a diversas regiones del mundo, debido a la emigración de ciudadanos de estos países en especial Israel, Siria, Líbano y en Estados Unidos en las ciudades de Los Ángeles, Nueva Cork, Miami, Houston, San Francisco y otras. En donde radican cerca de 500.000 argentinos que consumen el mate igual en que su país natal.

El mate contiene varios minerales, incluyendo fósforo, hierro, calcio y vitaminas $\mathrm{C}, \mathrm{BI}$ y $\mathrm{B}$ 2 ; contiene entre el 0,8 y 2,0\% de cafeína; entre el 7,0 y $14,0 \%$ de tanino. También se toma para promover la pérdida de peso y como estimulante para el sistema nervioso, diurético y antirreumático².

En 1997 Uruguay consumía anualmente entre 6 a $8 \mathrm{~kg}$. de yerba mate por persona. En el mismo período el consumo en Argentina era de $5,14 \mathrm{~kg}$. por persona. Se estima que en el sur de Brasil el $70 \%$ de los hombres y el $50 \%$ de las mujeres, consumen mate diariamente.

Se considera motivo de debate el papel de la yerba mate como factor de riesgo para el desarrollo del cáncer en el tracto superior del área digestiva. Estudios clínicos y epidemiológicos han encontrado desde un moderado hasta un alto riesgo de neoplasias en la parte superior del área digestiva, entre los consumidores de mate. La incidencia del cáncer en el aparato digestivo superior en Argentina, Uruguay y el sur del Brasil es muy alto y sigue en aumento ${ }^{3,4}$.

El principal problema para evaluar los efectos del consumo de yerba mate como un agente carcinógeno es la dificultad para obtener los controles apropiados de otros factores de riesgo que pueden alterar los resultados, como el alcohol y el tabaco.

En Brasil la incidencia del cáncer de laringe está entre los más altos del mundo. En un análisis comparativo los consumidores de yerba mate contraen 2.1 veces más cáncer del aparato digestivo superior comparado a los que nunca consumen yerba mate. En relación al consumo de mate se ha estudiado el posible impacto negativo de la utilización de agua a temperaturas muy elevadas, sin hallarse datos que corroboren su acción.

En Uruguay el cáncer bucal y orofaringe ha multiplicado cinco veces la incidencia debido al consumo de yerba mate ${ }^{3}$. El porcentaje de muertes provocado por estas enfermedades es de I4.5 por cada 100.000 entre los hombres y de 3,8 cada 100.000 entre las mujeres. Un análisis comparativo determinó que los consumidores de mate diario fueron encontrados 1.9 veces más propensos a contraer cáncer de la región orofaringea, comparados con los que no toman mate ${ }^{4}$.

En la boca, el daño por consumo de mate es proporcional a la temperatura. Por ello es importante señalar que la mayoría de los fumadores ingieren mate y/o café, potenciando aún más la relación y esto puede agravar la evolución de cualquier lesión oral existente. Del mismo modo, los alimentos calientes, picantes o muy condimentados constituyen un factor etiológico significativo en todo el tubo digestivo. Aunque el tiempo que actúan sobre los tejidos bucales depende del modo de masticación y la cantidad de alimentos que se ingieren, está demostrado que este tipo de dieta irritante puede alterar a las células bucales ${ }^{5}$.

La evidencia en la literatura sugiere que el consumo de yerba mate es cancerígeno y desempeña un papel en el desarrollo de los cánceres de la cavidad oral, faringe, laringe y esófago ${ }^{6}$.

El mecanismo exacto de la carcinogénesis de la yerba mate es desconocido pero se han sugerido dos factores etiológicos, el térmico y el químico ${ }^{7}$.

Por la complejidad del tema y de acuerdo con los adelantos de la ciencia y la tecnología en el campo de la Oncología, el objetivo fue determinar la acción térmica del mate y el hábito de fumar como factores predisponentes en la producción de cáncer en la cavidad bucal ${ }^{8}$.

\section{Materiales y Método}

Este trabajo se llevó a cabo en la Cátedra de Clínica Estomatológica, Servicio de Estomatología y Hospital Odontológico Universitario de la Facultad de Odontología de la Universidad $\mathrm{Na}$ cional del Nordeste.

La investigación fue de tipo observacional de corte transversal. Se incluyeron en la muestra 100 individuos de ambos sexos, cuyo rango de edades oscilaron entre 20 y 60 años, consumidores de mate, que residían en las provincias de Corrientes, Chaco y Formosa, que concurrieron voluntariamente para su atención en las clínicas, durante el período 2010 - 2014. 
Se formaron dos grupos de trabajo, un grupo constituido por cincuenta (50) individuos consumidores de mate caliente con bombilla metálica, y otro grupo de cincuenta (50) individuos fumadores y consumidores de mate caliente con bombilla metálica.

Se excluyeron de la muestra a individuos que padecían alteraciones sistémicas que cursaban con fiebre, procesos inflamatorios - infecciosos, trismo, dolores antálgicos que impedían un correcto examen bucal, así como, mujeres embarazadas, niños, discapacitados motrices o mentales.

Se explicaron cada una de las instancias previstas en el proyecto a los participantes y luego se solicitó el consentimiento informado para formar voluntariamente parte de la muestra.

En principio, se efectuaron exámenes bucales siguiendo la técnica de examen bucal de Grinspan, D. y se confeccionaron historias clínicas estomatológicas ${ }^{7}$.

Se citó en forma programada a los pacientes para tomar las muestras. A cada paciente se le entregó un equipo de mate, conformado por un termo con agua a temperatura de $84^{\circ}, 84^{\circ}$ - $86^{\circ}, 86^{\circ}-88^{\circ}, 88^{\circ}-90^{\circ}$, según la elección del paciente, un mate con yerba de buena calidad y de sabor suave, junto con a una bombilla metálica. Cada individuo preparó de manera habitual su mate y antes de la ingesta, se registraron en planillas, la temperatura del agua del termo con un termómetro de mercurio y luego la temperatura de los sectores de mucosa bucal a estudiar: semimucosa labial inferior (lugar donde apoya la bombilla metálica), zona retrocomisural de la mucosa yugal y a los lados del rafe medio del paladar duro con un termómetro digital Láser marca OMRON.

Antes de consumir el primer mate también se obtuvieron muestras a través de citología exfoliativa de las zonas de interés las que fueron analizadas histopatológicamente teniendo en cuenta las siguientes referencias según el grado de alteración celular de la mucosa según Stern Modificada (Bhaskar, 1989) 8, 9, 10.

MON: mucosa oral normal. Sin cambios inflamatorios.

I/L: Inflamación leve. Presencia de discreta alteración celular en el epitelio.

I/M: Inflamación moderada. Presencia de dis- creta alteración celular en el epitelio y cambios subepiteliales.

I/S: Inflamación severa. Presencia de moderada alteración celular en el epitelio y cambios subepiteliales.

El paciente iniciaba el consumo de mate y a los 60 minutos se efectuaban las tomas de temperatura de la mucosa bucal en las mismas zonas antes estudiadas y con su correspondiente examen citológico.

Cada muestra obtenida fue fijada en alcohol $96^{\circ}$ e identificada con un código correspondiente al número de historia clínica, año y procedencia, para su envío al Laboratorio de Anatomía Patológica de la de Facultad de Odontología de la Universidad Nacional del Nordeste.

\section{Resultados}

Del total de la muestra estudiada el $56 \%$ fue del sexo femenino mientras que el $44 \%$ fueron masculinos con una edad promedio de entre 31 y 35 años. Del total de mujeres, el $42,5 \%$ fumaban cigarrillos diariamente y con respecto a los hombres el $57,5 \%$ eran fumadores.

A través del Test de Pearson, se evaluó la correlación entre la temperatura de las mucosas bucales después del mate y la temperatura del agua del termo con la que se iniciaba el consumo, encontrándose $r=0,33$, P-valor $=0,00 \mathrm{I}$ habiendo una relación significativa. Por tanto, al elevarse la temperatura del agua del mate, se elevaban las temperaturas de las mucosas bucales. Las categorías establecidas de la temperatura de agua del termo antes de iniciar el consumo de mate fueron: menor a $84^{\circ}, 84^{\circ}-86^{\circ}, 86^{\circ}-88^{\circ}$ y $88^{\circ}-90^{\circ}$. Mientras que las categorías analizadas de la temperatura de las mucosas bucales después de la ingesta fueron: menor a $45^{\circ}, 45^{\circ}-55^{\circ}$ y $55^{\circ}-65^{\circ}$.

Con respecto a la relación entre la temperatura de las mucosas bucales antes del consumo y la temperatura del agua, fue de $r=-0,12 \mathrm{P}$-valor $=0,239$ I, no existiendo relación significativa.

Se observó que la relación entre grado de inflamación después del consumo de mate y zonas topográficas de la mucosa bucal variaba según su localización, observándose mayor grado de normalidad en la mucosa yugal (60 casos), luego en el labio inferior (22 casos) y en el paladar duro (18 casos). Mientras que se encontró 
inflamación leve en mayor cantidad en el paladar duro 24 casos, así como modera y severa en 4 y 8 casos respectivamente.

Con la prueba de Chi Cuadrado $=0,0102$ se estableció que la intensidad de la inflamación en el epitelio bucal registrada según diferentes criterios histopatológicos, está directamente relacionada con la zona topográfica estudiada, en este caso indicando mayor grado de severidad en el paladar duro.

Con respecto a la relación entre el consumo diario de mate y el hábito de fumar. Se com- probó que los pacientes fumadores, antes de iniciar el consumo de mate, poseían un $70 \%$ de células inflamatorias leves y un $30 \%$ normales en la mucosa bucal, mientras que si no fumaban sólo el $20 \%$ eran células inflamatorias leves y el $80 \%$ normales. Al terminar el consumo de mate, los pacientes fumadores registraron células inflamatorias severas (39\%), moderadas (27\%) y leves $(27 \%)$ y un $7 \%$ normales en el epitelio bucal, mientras que los no fumadores sólo presentaron células inflamatorias leves (45\%) y moderadas $(4 \%)$ y un $51 \%$ normales.

Tabla I. Temperatura del agua y su relación con estudios citológicos de las mucosas bucales.

\begin{tabular}{|c|c|c|c|c|}
\hline Citologías/Temperaturas & $84^{\circ} \mathrm{C}$ & $84-86^{\circ} \mathrm{C}$ & $86-88^{\circ} \mathrm{C}$ & $88-90^{\circ} \mathrm{C}$ \\
\hline Mucosa Oral Normal & 7 & 67 & I & 89 \\
\hline Inflamación leve & 0 & 71 & 39 & 127 \\
\hline Inflamación moderada & 0 & 6 & 37 & 45 \\
\hline Inflamación severa & 0 & 0 & 39 & 39 \\
\hline Total & 7 & 144 & 116 & 300 \\
\hline Estadístico & \multicolumn{2}{|c|}{ Valor gl } & \multirow[b]{2}{*}{12} & \multirow[b]{2}{*}{$<0,000$ । } \\
\hline Chi Cuadrado Pearson & & 165,72 & & \\
\hline
\end{tabular}

Tabla 2. Temperatura de las mucosas bucales después del consumo de mate y su relación con resultados citológicos.

\begin{tabular}{|l|c|c|c|c|}
\hline \multicolumn{1}{|c|}{ Citologías/Temperaturas } & $45^{\circ} \mathbf{C}$ & $45-55^{\circ} \mathbf{C}$ & $55-65^{\circ} \mathbf{C}$ & Total \\
\hline Mucosa Oral Normal & 51 & 22 & 16 & 88 \\
\hline Inflamación leve & 35 & 52 & 40 & 127 \\
\hline Inflamación moderada & 11 & 23 & 11 & 45 \\
\hline Inflamación severa & 11 & 15 & 13 & 39 \\
\hline Total de muestras & 108 & 112 & 80 & 300 \\
\hline
\end{tabular}

\begin{tabular}{lllr} 
Estadístico & Valor & gl & P \\
\hline Chi Cuadrado Pearson & 26,96 & 6 & $<, 000$ I
\end{tabular}


Figura I. Resultados de estudios citológicos antes del consumo de mate caliente en pacientes fumadores.

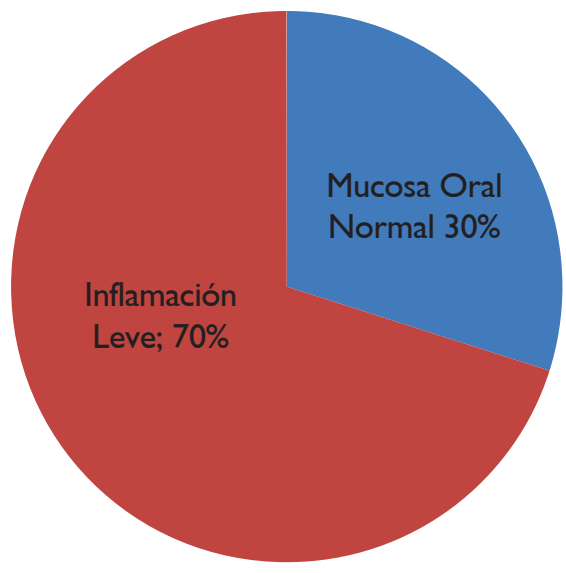

\section{Discusión}

El conocimiento y el control de los factores de riesgo del cáncer bucal son de gran importancia para la práctica estomatológica y médica.

Se considera factor de riesgo cualquier elemento que pueda aumentar las posibilidades de una persona para desarrollar alguna enfermedad. Aun cuando los factores de riesgo en algún momento desencadenen una enfermedad, éstos no necesariamente causan la enfermedad. Muchos pacientes con uno o más factores de riesgo nunca desarrollan cáncer mientras otros, lo desarrollan sin tener factores de riesgo conocidos ${ }^{11,12,13}$.

Se describen los principales factores exógenos como el tabaquismo, el consumo de alcohol y la mala higiene bucal, como capaces de originar lesiones precursoras y malignas en la boca. Estos carcinógenos causan daño tisular crónico que se manifiestan en edades avanzadas, por lo que dependen de la temporalidad y pueden potenciar su acción ante traumas crónicos o irritantes bucales térmicos ${ }^{14,15,16,17 .}$

El presente trabajo estudió el efecto carcinogénico de la acción térmica del agua del mate en la cavidad bucal, ya que la bibliografía consultada sólo relataba, que la bebida de yerba mate es un factor de riesgo para el cáncer de laringe, esófago y orofaringe, siendo reconocido su factor térmico, sin ser identificados al momento los mecanismos de la carcinogénesis ${ }^{18,19,20,21}$.
Figura 2. Resultados de estudios citológicos después del consumo de mate caliente en pacientes fumadores.

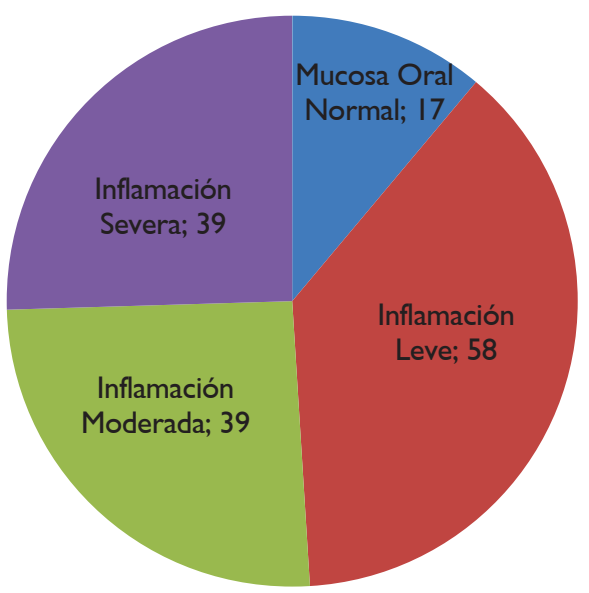

El Dr. Stefany (Uruguay) considera que el consumo de yerba mate multiplica por cinco la incidencia en el cáncer orofaringeal, con un efecto multiplicador de los consumidores de tabaco negro, vino y yerba mate. En este sentido este trabajo coincide con la afirmación que el consumo de mate potencia la acción nociva del tabaco sobre las mucosas orales.

El Dr. Reggia (Uruguay) luego de un estudio realizado en Montevideo sobre el desarrollo de cáncer de boca, lengua y encías afirma que los resultados muestran un exceso de cáncer de boca en los consumidores de yerba mate, sin embargo, se han encontrado diferencias en cuanto a la localización y se considera que aún faltan realizar estudios que demuestren la acción del mate como factor de riesgo en el cáncer bucal ${ }^{22}$.

Se coincide con el artículo de la Agencia Internacional de Investigación de Cáncer (IARC), que en 199I ya evaluaba el riesgo carcinogénico del mate a través de la insuficiente evidencia de la carcinogenicidad de la bebida de yerba mate en humanos, no existiendo información disponible acerca del consumo de mate frío. Sin embargo, la IARC clasificó la bebida de yerba mate caliente como un probable carcinógeno para los humanos ${ }^{23}$.

El cáncer bucal es una patología que ocupa 3\% aproximadamente de todos los cánceres localizándose en labios, lengua, piso de boca, muco- 
Figura 3. Resultados de estudios citológicos antes del consumo de mate caliente en pacientes no fumadores.

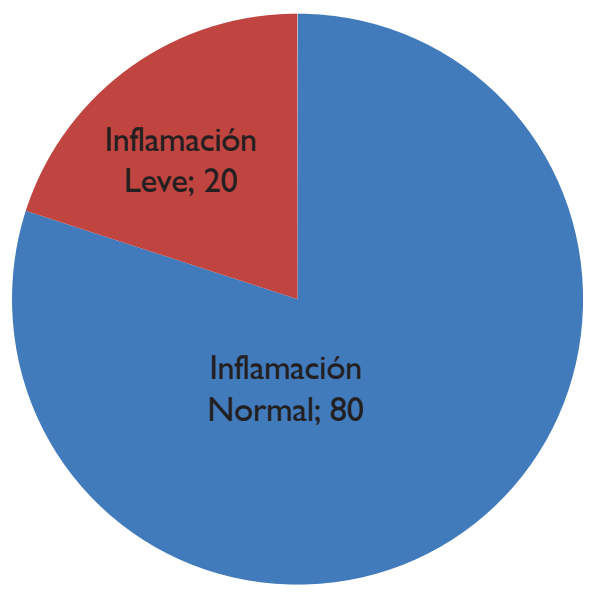

sa bucal, carrillos, trígono retromolar y paladar duro, de acuerdo con la Sociedad Americana del Cáncer (ACS). I-4 A partir del trabajo realizado, se observó mayor incidencia de lesiones inflamatorias en el paladar duro provocadas por el consumo de mate caliente con bombillas metálicas.

En la investigación desarrollada centrada en la asociación entre la acción térmica del mate y el hábito de fumar, se evidenció que existe correlación positiva entre ambos factores exógenos $^{24,25,26}$.

Se coincide con los autores en las diferencias de género existentes en relación a roles de la práctica de hábitos tóxicos siendo más comunes en el masculino, por eso existe un criterio tradicional de que el cáncer bucal es más frecuente en los hombres comparados con las mujeres; pero realmente no se han encontrado diferencias estructurales que apunten a que un sexo sea más vulnerable que el otro ${ }^{25-27}$.

\section{Conclusiones}

La zona topográfica más afectada en la cavidad bucal en pacientes que consumen mate caliente y poseen el hábito de fumar es el paladar duro.

Se observó un mayor porcentaje de hombres fumadores con respecto a las mujeres en una relación $2: 1$.
Figura 4. Resultados de estudios citológicos después del consumo de mate caliente en pacientes no fumadores.

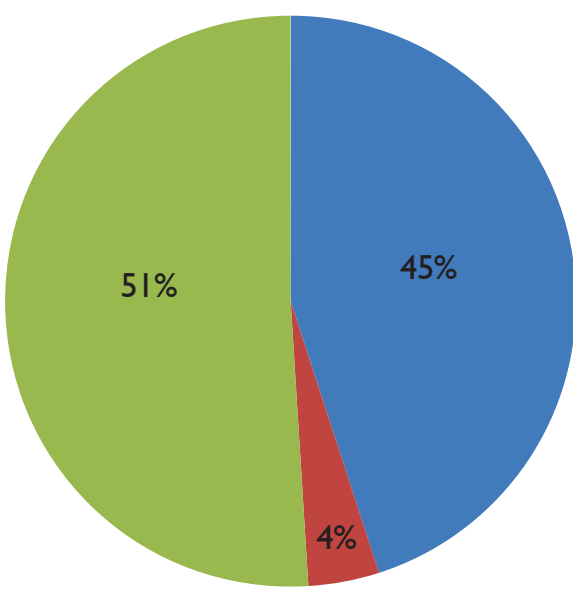

Inflamación Leve

Inflamación Moderada

Normal

Al elevarse la temperatura del agua del mate, se elevaron las temperaturas de las mucosas bucales. Con respecto a la relación entre la temperatura de las mucosas bucales antes del consumo y la temperatura del agua, no existió relación significativa.

Existe una significación estadística entre los resultados histopatológicos de las mucosas bucales y la temperatura del agua del mate. Cuando la temperatura del agua del mate antes del inicio de su consumo fue de 88 a $90^{\circ}$ se observó en su gran mayoría una inflamación leve en el epitelio de las mucosas; este fenómeno no fue observado cuando la temperatura del agua del mate disminuyó, encontrándose un epitelio con características de normalidad.

Se obtuvo una significación estadística entre la citología y la temperatura de las mucosas después del consumo de mate caliente. Se observó que si la temperatura de las mucosas bucales después del consumo de mate era menor a 45 grados, el porcentaje de células normales era $47 \%$ mientras que si la temperatura era de 55 a 65 grados el porcentaje de células normales era $20 \%$. 


\section{Bibliografía}

I. Pharynx (monografía en soporte electrónico). Edge SB, Byrd DR, Compton CC, et al. Cancer Staging Manual. 7th ed. New York, NY: Springer; 2010.

2. Goldenberg D, Golz A, Joachims HZ. La bebida yerba mate: un factor de riesgo para cáncer de cabeza y cuello. Taringa Inteligencia Colectiva (publicación periódica en línea) 201I (citada mayo 2014). Disponible en: URL: http://www. taringa.net/posts/info/ / 0628299/Yerba-Mateproduce-riesgos-adicionales-de-contraer-cance. html

3. Sewram, V, De Stefani E, Brennan P, Boffetta P. Maté consumption and the risk of squamous cell esophageal cancer in Uruguay. Cancer Epidemiol Biomarkers Prev. 2003 Jun; I2 (6): 508- I3. (citada mayo 2014). Disponible en: http://www.ncbi. nlm.nih.gov/pubmed/I28I4995

4. Loria D, Barrios E, Zanetti R. Cancer and yerba mate consumption: a review of possible associations. Rev Panam Salud Pública. 2009; 25 (6): 530-539. (citada mayo 20l4). Disponible en: http://www.scielosp.org/pdf/rpsp/v25n6/ v25n6al0

5. Rojas M H, Santana Garay J C, González Docando Y E, Borroto Alcorta D C. Factores de riesgo del cáncer bucal. Disponible en: http://bvs. sld.cu/revistas/mciego/voll3_0l_07/articulos/ al2_vl3_0107.htm Acceso: 10/02/2016

6. Gallegos Hernandez JF. El cáncer de cabeza y cuello. Factores de riesgo y prevención. Cir Ciruj. 2006; 74 (4): 287-93. (citada mayo 2014). Disponible en: http://www.medigraphic.com/ pdfs/circir/cc-2006/cc064m.pdf

7. Grinspan D. Enfermedades de la Boca, Semiología, Patología, Clínica y terapéutica de la Mucosa Bucal. Buenos Aires, 1976.

8. Jemal A, Thomas A, Murray T, et al. Cáncer Statistics. CA Cancer J Clin 2002; 52: 6-7. (citada mayo 2014). Disponible en: http://www.ncbi. nlm.nih.gov/pubmed/I |8|4064.

9. Bogumiła Szyszka-Charewicz MD, Katarzyna Pudełek MD, Tomasz Skóra MD, Jan Skołyszewski MD. Is Radiotherapy for Early Laryngeal Cancer in Elderly a Safe and Effective Treatment Method. Austral - Asian Journal of Cancer. 20I I; 10 (4): 255-6I. (citada mayo 20I4). Disponible en: URL: RadiotherapyforEarlyLaryngealCanerElderlyaSafeandEffectiveTreatmentMethod(I).pdf
10. Jones KR, Lodge-Rigal RD, Reddick RL, Tudor GE, Shockley WW. Prognostic factors in the recurrence of stage I and II squamous cell cancer of the oral cavity. Archives of Otolaryngol, head Neck Surg. 1992; II 5 (5): 483-5. (citada mayo 2014). Disponible en: http://www.ncbi.nlm.nih. gov/pubmed/I57 I I I6.

II. Jee SH, Samet JM, Ohrr H, Kim JH, Kim IS. Smoking and cancer risk in Korean men and women. Cancer Causes Control. 2004 May; I5 (4): 34I-8.

12. Peña González A, Arredondo López M, Vila Martínez L. Comportamiento clínico y epidemiológico del cáncer de cavidad oral. Rev Cuba Estomatol. 2006; 43 (I). (citada mayo 20I4). Disponible en: http://www.bvs.sld.cu/revistas/ est/vol43_I_06/est03106.htm.

13. Accortt NA, Waterbor JW, Beall C, Howard G. Cancer incidence among a cohort of smokeless tobacco users (United States). Cancer Causes Control. 2005 Nov; I6 (9): I I07-I4. (citada mayo 2014). Disponible en: http://www.ncbi. nlm.nih.gov/pubmed/I6184477.

14. Weber AL, Romo L, Hashmi S. Malignant tumors of the oral cavity and oropharynx: clinical, pathologic, and radiologic evaluation. Neuroimaging Clin N Am. 2003; 13 (3): 443-64. (citada mayo 2014). Disponible en: http://www.ncbi. nlm.nih.gov/pubmed/I463 I684.

I5. Andersson G, Vala EK, Curvall M. The influence of cigarette consumption and smoking machine yields of tartar and nicotine on the nicotine uptake and oral mucosal lesions in smokers. J. Oral Pathol Med. I 997 Mar; 26 (3): I I7-23. (citada mayo 2014). Disponible en: http://www.ncbi. nlm.nih.gov/pubmed/9083935.

16. Verra F, Zabert G, Ferrante D, Morello P, Virgolini M. Consumo de tabaco en estudiantes de educación secundaria de Argentina. Rev Panam Salud Publica. 2009; 25 (3): 227-33. (citada mayo 2014). Disponible en: http://www.msal.gov.ar/tabaco/images/stories/institucional/pdf/consumode-tabaco-2002.pdf

17. Bello Santos P, Reyes Velásquez JO, Véjar Alba I. El papel del Cirujano Dentista en la detección oportuna del cáncer bucal. Presentación de un caso. Med Oral. 200 I; 3 (2): 65-68. (citada mayo 2014). Disponible en: http://connection.ebscohost.com/c/articles/2557623 I/el-papel-del-cirujano-dentista-en-la-detecci-n-oportuna-del-cncer-bucal-presentaci-n-de-un-caso 
18. Holmstrup, P. The controversy of a premalignant potential of oral lichen planus is over. Oral surgery, oral medicine, oral pathology. 1992; 73 (6): 704-706. (citada mayo 2014). Disponible en: http://www.ncbi.nlm.nih.gov/pubmed/I 437040

19. Takagi M, Kayano T, Yamamoto H, et al. Causes of oral tongue cancer treatment failures: Analysis of autopsy cases. Cancer. 1992; 69 (5): 108I-7. (citada mayo 2014). Disponible en: http://onlinelibrary.wiley.com/doi//0.1002/ cncr.2820690502/pdf

20. Franceschi D, Gupta R, Spiro RH, et al. Improved survival in the treatment of squamous carcinoma of the oral tongue. Am J Surg. 1993; 166 (4): 360-5. (citada mayo 20I4). Disponible en: http:// www.ncbi.nlm.nih.gov/pubmed/82 I4293?dopt=

2I. Cole PH, Rodu B. Analytic Epidemiology: Cancer Causes. Epidemiology of Cancer. En: (Guías en soporte electrónico) De Vita VT, Hellman S, Rosemberg SA. Cancer. Principles \& Practice of Oncology. Filadelfia: Lippincott Williams \& Wilins. 200I: 24I-252. (citada mayo 20I4). Disponible en: http://joh.sanei.or.jp/pdf/E49/ E49_I_09.pdf

22. Prácticas de Estomatología. La Habana: Editorial Ciencias Médicas. 2003:472-7.

23. Hermida Rojas M, Santana Garay JC, Hernández Díaz MJ, et al. El estilo de vida en pacientes con cáncer bucal. Rev MEDICIEGO. 2005; I I (2). (citada mayo 2014). Disponible en: http://www.bvs. sld.cu/revistas/mciego/voll3_0I_07/articulos/ al2_v13_0107.html

24. Goldemberg D. Mate: a risk factor for oral and oropharyngeal cancers. Oral Oncol. 2002; 38(7): 646-9. (citada mayo 2014).Disponible en: http:// www.ncbi.nlm.nih.gov/pubmed//21674I7.

25. Oliveira LR, Ribeiro-Silva A, Zucoloto S. Perfil da incidência e da sobrevida de pacientes com carcinoma epidermóide oral em uma população brasileira. J Bras Patol Med Lab. 2006;42(5):385392. (citada mayo 2014). Disponible en: http:// www.scielo.br/pdf/jbpml/v42n5/a 10v42n5.pdf

26. Brandizzi D, Chuchurru JA, Lanfranchi Tizeira HE, Cabrini RL. Análisis de las características epidemiológicas del cáncer bucal en la ciudad de Buenos Aires. Acta Odontol Latinoam. 2005; 18 (I): 3I-35. (citada mayo 20I4). Disponible en: http://bases.bireme.br/cgibin/wxislind.exe/iah/ online/?lsisScript=iah/iah.xis\&src=google\&base= LILACS\&lang=p\&nextAction=Ink\&exprSearch= $4|788|$ \&indexSearch=ID 